\title{
DRIVER DISTRACTION AND RELIANCE: ADAPTIVE CRUISE CONTROL IN THE CONTEXT OF SENSOR RELIABILITY AND ALGORITHM LIMITS
}

\author{
Bobbie D. Seppelt, Monica N. Lees, John D. Lee \\ Department of Mechanical and Industrial Engineering \\ Center for Computer-Aided Design \\ University of Iowa \\ Iowa City, Iowa, USA \\ E-mail: bseppelt@engineering.uiowa.edu
}

\begin{abstract}
Summary: This study investigated how system failures influenced drivers' reliance on Adaptive Cruise Control (ACC). A medium-fidelity driving simulator was used to evaluate the effect of driving condition (traffic, rain) and automation (manual control, ACC) on headway maintenance and brake response. In conditions of rain, the signal continuity of the ACC sensors was degraded and in conditions of heavy traffic, the braking limits of the ACC system were exceeded. Dependent variables included response time to lead vehicle (LV) braking, number of collisions, and both time headway (THW) and time-to-collision (TTC) at instant of the brake response. Throughout the drive, a continuous (forced-paced) secondary task was introduced to determine how an in-vehicle task interacted with ACC reliance. Results showed that the failure type influenced driver's reliance on ACC with drivers relying more on ACC in traffic periods than in rain periods. ACC appeared to offer a safety benefit when drivers were distracted with complex mental tasks in periods of heavy traffic.
\end{abstract}

\section{INTRODUCTION}

In 2003, over six million motor vehicle crashes occurred in the United States. Rear-end collisions represented approximately $30 \%$ of the total crashes and resulted in 2,302 fatalities and approximately one million injuries (Lee et al., 2002; NCSA, 2003). Two factors are primarily responsible for rear-end collisions: (1) inattention and (2) unsafe following distances (Dingus et al., 1997). Adaptive Cruise Control (ACC) provides a potential safety benefit in helping drivers to maintain a constant speed and headway distance (Davis, 2004). Specifically, ACC adjusts the brake and throttle to maintain a constant headway from any vehicle that intrudes upon the path of the driver's vehicle. Previous studies, however, have shown degraded performance when ACC has failed (Nilsson, 1995; Stanton \& Young, 1998). But these studies do not address the complexity of automation reliability. The consequence of automation failures depend on the type of failure and the context (Lee \& See, 2004).

ACC is a convenience system and as such, drivers must refrain from becoming dependent on the system for braking response. For ACC to be effective, drivers need to understand the capabilities of ACC, which depend on both braking and sensor limitations. Based on this understanding, they must be able to intervene when the situation exceeds ACC capabilities. However, drivers have difficulties in understanding how ACC functions (Stanton \& Marsden, 1996). As a result, they inappropriately rely on the system. For instance, Nilsson (1995) found that when approaching a queue of vehicles, drivers failed to intervene because they believed that ACC could effectively respond to the situation. 
The current experiment investigated how system failures influenced drivers' reliance on ACC. Two failure types were introduced within specific driving contexts to determine their effect on ACC reliance. Further, a secondary task was included to determine the effect of driver distraction on headway maintenance performance. The results are hypothesized to show that reliance on ACC, coupled with simple drives (i.e., straight road), will lead drivers to disengage from the driving task and delay responses to changes in lead vehicle (LV) speed. The sensitivity of the system's braking response to LV decelerations is expected to mitigate the increase in brake response time, leading to a net safety benefit of using ACC over manual control. This research investigates the ability of drivers to intervene and resume manual control, as well as how different types of system failures influence reliance on the ACC system.

\section{METHOD}

\section{Participants}

Eight males and eight females between the ages of 25 and $40(M=30.1, S D=4.6)$ participated in the study. All participants were native English speakers with active driver's licenses. Participants were recruited as volunteers and paid $\$ 15$ per hour for their participation, with a total $2 \mathrm{~h}$ participation time. Participants received additional compensation (up to \$10) if they performed well on the secondary task.

\section{Driving Environment Overview}

Data were collected using a medium-fidelity, fixed-base GlobalSim Corporation Vection Research Simulator (VRS). The simulator utilizes a 1992 Mercury Sable cab equipped with a 50degree field of view, full instrumentation with functional gauges, a force feedback steering wheel, and a surround-sound audio system. The fully-textured graphics are generated by state-ofthe-art PC hardware, which delivers a $60-\mathrm{Hz}$ frame rate at 1024 x 768 resolution. Data were collected at $60 \mathrm{~Hz}$.

The driving environment consisted of alternating sections of two-lane rural and four-lane urban roadways. No curves were included to create a low-demand environment and to promote disengagement from the driving task. Participants drove the roadway in both directions, encountering oncoming traffic at a rate of three to five cars per minute.

\section{Driving Task}

Each participant drove two 18-minute drives, one without ACC and one in which ACC was available. When ACC was not in use, drivers were instructed to maintain an approximate $1.5 \mathrm{~s}$ following distance from a LV, which varied in speed throughout the drive. Within each drive, drivers experienced two 3-minute periods of both traffic and rain. For traffic and rain periods, a decrease in the LV's velocity was defined as a braking event; there were 11 braking events per traffic period and 7 braking events per rain period, spaced 12-15 and 24-28 seconds apart, respectively. In the traffic periods, the LV velocity varied according to a Modulo function, defined as a sharp velocity decrease followed with a gradual increase to the original level. In the rain periods, the LV velocity followed a seemingly random pattern defined by the sum of two sine waves. 


\section{Adaptive Cruise Control (ACC)}

The ACC system operated when the vehicle was traveling between $20 \mathrm{mph}(32 \mathrm{~km} / \mathrm{h})$ and 85 $\mathrm{mph}(137 \mathrm{~km} / \mathrm{h})$. If no vehicle was detected in front of the participant vehicle, ACC operated as would a normal cruise control system, accelerating to a set speed of $55 \mathrm{mph}$. If a slower moving vehicle was in front of the participant vehicle, the vehicle speed was modulated by the ACC algorithm to maintain a $1.5 \mathrm{~s}$ time headway (THW) setpoint. The ACC system applied a maximum of $33 \%$ of the vehicle's braking power, or $0.28 \mathrm{~g}$.

ACC Braking Algorithm Limits. In the traffic periods, the magnitude of each LV velocity change increased over time, peaked at 90 seconds, and then decreased in a symmetrical fashion. Traffic density influenced the LV velocity. As the braking of the LV became more severe, the $0.28 \mathrm{~g}$ braking limit of ACC was exceeded, with the ACC system requiring longer and more intense braking, compromising the ability of the ACC to maintain a $1.5 \mathrm{~s}$ headway.

ACC Sensor Degradation. In the rain periods, the degree of fog and rain increased over time, peaked at 90 seconds, and then decreased symmetrically. Rain degraded the ability of the radar to detect vehicles ahead, masking the sensors and causing them to fail temporarily. As the intensity of the rain increased, the sensors failed for longer periods and failed more frequently. The magnitude and duration of the sensor failures during the rain periods paralleled the increasing and decreasing effects of the traffic. The momentary failure of the sensors to detect vehicles ahead compromised the ability of the ACC to maintain a $1.5 \mathrm{~s}$ headway.

\section{Secondary Task}

Throughout the drive, a continuous (forced-paced) secondary task required listening and verbally responding to messages related to upcoming restaurants. Each message presented information pertaining to the average entrée cost, quality rating, and wait time for three restaurants. A total of six messages per drive were presented, with each message followed by a series of six questions that were based on the information given within the message. Complexity of response was dictated by the number of criteria used to identify a restaurant.

\section{Experimental Design}

The experiment combined two levels of scenario type (i.e., failure type), rain and traffic, and two levels of automation, no ACC and ACC control, in a 2x2 within-subjects factorial design. The order of conditions was counterbalanced using a Latin square design.

\section{Procedure}

Upon arriving, participants completed an informed consent form and then drove a short drive to acclimate them to the driving simulator controls and environment. Participants drove an additional 5-minute practice drive before each of the two experimental drives. For the ACC drive, participants were advised that $\mathrm{ACC}$ be used only if the weather and traffic conditions allowed safe driving at a steady speed. Following the experimental drives, participants were debriefed and compensated. 


\section{RESULTS}

Results are described according to driving and secondary task performance. The first section addresses the effects of automation level, and failure type on measures of driving task performance including brake response time (RT), time headway (THW) at point of brake response, time-to-collision (TTC) at point of brake response, number of collisions, and percent reliance. The second section addresses the effects of the same independent variables, with the addition of message complexity, on headway maintenance performance while engaged in the secondary task. Note that the ACC drive was divided into engaged and not engaged portions to capture differences in driving and secondary task performance with manual and ACC control. The analyses were performed using SAS 9.0 software; $F$ statistics are reported from repeated measures ANOVAs.

\section{Driving Task Performance}

Brake Response. Brake RT is defined as the time from the point the LV begins to decelerate to when a driver depresses the brake more than $5 \%$ of the total brake pedal range. During the traffic periods, in which ACC braking capacity was exceeded, mean brake RT showed a trend towards longer response times when ACC was engaged $(t(28)=2.04 ; p=0.0513)$. However, the braking response of ACC allowed drivers to wait longer to respond to LV braking behavior, resulting in both a longer THW and TTC at brake response during ACC control compared to manual control $(F(2,27)=10.61, p=0.0004 ; F(2,22)=7.61, p=0.003$, respectively; see Table 1$)$. In addition, significantly fewer collisions occurred during ACC control compared to manual control $\left(\chi^{2}(1)=9.77, p=0.002\right.$; see Table 1$)$. Thus, for traffic situations, a net safety benefit in using ACC was evident.

Table 1. Summary results of Brake RT, THW at Brake, TTC at Brake, and Collisions by Automation Level for Rain and Traffic periods

\begin{tabular}{|c|c|c|c|c|c|c|}
\hline & \multicolumn{6}{|c|}{ Automation level } \\
\hline & \multicolumn{2}{|c|}{ No ACC (Manual) } & \multicolumn{2}{|c|}{ ACC engaged } & \multicolumn{2}{|c|}{ ACC not engaged } \\
\hline & M & SE & M & $\mathrm{SE}$ & M & SE \\
\hline \multicolumn{7}{|l|}{ Traffic periods } \\
\hline Brake RT (s) & $2.13^{* *}$ & 0.16 & $3.22^{* *}$ & 0.86 & 2.17 & 0.19 \\
\hline THW at brake(s) & $1.22^{*}$ & 0.10 & $3.29^{*}$ & 0.82 & 1.48 & 0.12 \\
\hline TTC at brake(s) & $4.38^{*}$ & 0.41 & $7.37^{*}$ & 1.01 & 4.58 & 0.52 \\
\hline Collisions & \multicolumn{2}{|c|}{$8^{*}$} & \multicolumn{2}{|c|}{$0^{*}$} & \multicolumn{2}{|c|}{1} \\
\hline \multicolumn{7}{|l|}{ Rain periods } \\
\hline Brake RT (s) ${ }^{1}$ & 8.42 & 0.99 & 5.48 & 2.59 & 6.62 & 1.01 \\
\hline THW at brake(s) & 1.55 & 0.15 & 1.87 & 0.37 & 2.36 & 0.35 \\
\hline TTC at brake(s) & 8.13 & 1.31 & 9.75 & 3.05 & 6.95 & 0.68 \\
\hline Collisions & \multicolumn{2}{|c|}{3} & \multicolumn{2}{|c|}{1} & \multicolumn{2}{|c|}{3} \\
\hline
\end{tabular}

* A significant effect at $\mathrm{p}<0.01$.

** A marginally significant effect at $\mathrm{p}<0.07$.

${ }^{1}$ The large brake RTs are due to the gradual brake events, which lasted $\sim 12$ seconds each. 
During the rain periods, in which ACC sensor reliability varied, drivers' brake response did not significantly differ between manual and ACC use $(t(25)=1.25, p=0.2216)$. Drivers maintained marginally longer THWs in the ACC compared to the manual drives $(F(2,17)=2.87, p=0.08$; see

Table 1). The sensor failures in the rain disabled the braking algorithms, thus no benefit of the system braking response existed with THW and TTC values $(F(2,17)=2.87, p=0.084$; $F(2,16)=1.09, p=0.358$, respectively). Given this, and the non-significance between manual and ACC control for the collision data $\left(\chi^{2}(1)=0.332, p=0.564\right)$, no net safety benefit was evident during rain periods.

Automation Reliance. To determine the degree to which drivers relied on ACC in rain and traffic periods, percent reliance across participants was calculated as a function of ACC competence for both rain and traffic conditions. The competence of the ACC varied inversely with the intensity of the rain and traffic conditions over the three-minute periods. During these periods ACC competence declined and then recovered, thus there are two values for each level. Competence is defined in terms of both range rate (e.g., velocity difference between the participant vehicle and the LV) and minimum THW (see Figure 1a and Figure 1b). A comparison of the range rate and minimum THW reliance profiles provides insight into the safety consequences of the two failure types. In the traffic periods, the ACC system compensates for the increased range rate in brake response as seen in the relatively unaffected THW. However, the THW precipitously declines as the ACC system is no longer able to match the lead vehicle deceleration. In the rain periods, the sensor failures result in comparable range rate and THW profiles, but decline in a more gradual fashion.
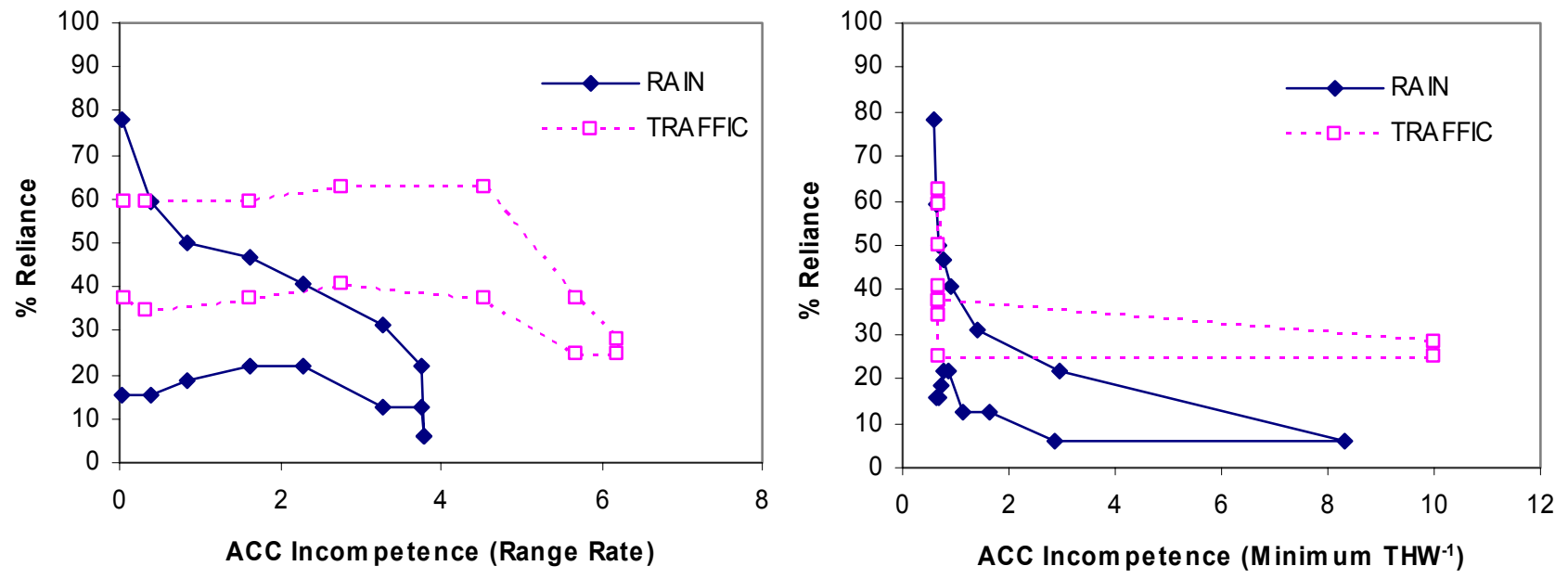

Figure 1. Percent reliance versus ACC incompetence by failure type; Rain $=$ sensor degradation; Traffic $=$ braking algorithm limits (a) Range Rate; (b) Minimum THW $^{-1}$

Plotting the ACC competence levels shows a non-linear profile of reliance on ACC, characterized by hysteresis, for both rain and traffic conditions $(t(10)=3.659, p=0.005$; $t(8)=3.224, p=0.013$, respectively). For a given competence level, drivers have a greater tendency to rely on ACC in traffic periods, where the braking algorithm limits limit ACC performance, than in rain periods, where the sensor failures limit ACC performance. Note, 
however, the larger hysteresis effect in rain (see Figure 1a). The distraction posed by the secondary task, when listening to messages or questions, did not affect reliance on ACC.

\section{Secondary Task Performance}

Secondary task data was broken down into two categories, responses made in less than three seconds and responses made in three seconds or greater. Due to limited responses made under ACC engagement during the rain portions of the drive, only traffic portions were analyzed; results are discussed according to headway maintenance during the secondary task.

There was a main effect of ACC on time headway, with those with ACC having a larger time headway than those without ACC $(M=1.50, S E=0.142 ; M=1.06, S E=0.103$, respectively), $F(2,184)=6.05, p=0.0028$. There was also a main effect of response time, with those responding in less than three seconds having a larger THW than those responding in three seconds or more $(M=1.55, S E=0.093 ; M=1.23, S E=0.115$, respectively), $F(1,174)=4.21, p<0.0417$. Finally, there was an interaction between ACC reliance and secondary task complexity, $F(2,198)=3.51$, $p<0.0317$. While answering simple questions, participants were able to maintain a $1.5 \mathrm{~s}$ THW. However, when task complexity and response time increased, THW substantially declined in manual control. These results suggest that ACC may improve the ability to maintain a larger THW, especially when drivers are responding to a complex secondary task.

\section{DISCUSSION}

Reliance on ACC led drivers to disengage from driving and increased drivers' response time to LV braking. In periods of traffic, the braking of the ACC compensated for the increased time it took for drivers to react to LV velocity changes, thus affording a net safety benefit. In periods of rain, the sensor failures undermined this safety benefit.

Drivers disengaged ACC more readily in rain conditions, and were less inclined to rely on the ACC even after it returned to previous levels of competence. In traffic, drivers disengaged ACC only after its competence had declined significantly. The reliance profile in both rain and traffic conditions showed hysteresis, with drivers relying on ACC more when the competence had begun to decline than after it had recovered; the reliance profiles suggest a mismatch in perceived and actual system function. Note that reliance on ACC is a dichotomous decision rather than a continuous response. Because ACC reliance is dichotomous, but its competence is continuous, a critical issue is the degree to which drivers' trust in ACC and vigilance to potential ACC failures corresponds to ACC capability (Lee \& See, 2004).

For the complex secondary task, the pattern of car following during distraction is influenced by the availability of ACC. When a driver is distracted, ACC improves drivers' ability to maintain a longer headway compared to when driving without ACC. In addition, as secondary task complexity and response time increase, the ability to maintain a safe headway distance is diminished in manual control. ACC appears to offer the greatest benefit when drivers are distracted with complex mental tasks. However, this effect depends on the degree of automationinduced disengagement in the driving task and the particular type of failure. Some ACC failures can undermine driving safety (Young \& Stanton, 2002). This study addressed two qualitatively 
different types of reliability and driver responses to changes in reliability over time and showed that the safety benefit of ACC and the reliance profile depends on failure type.

\section{ACKNOWLEDGMENTS}

This research was supported by Nissan Motor Co., Ltd., with special thanks to Erwin Boer, project coordinator.

\section{REFERENCES}

Davis, L.C. (2004). Effect of Adaptive Cruise Control Systems on Traffic Flow. Physical Review E, 69(6).

Dingus, T.A., McGehee, D.V., Manakkal, N., Jahns, S.K., Carney, C. \& Hankey, J.M. (1997). Human factors field evaluation of automotive headway maintenance/collision warning devices. Human Factor, 39(2): 216-229.

Hoedemaeker, M. (2000). Driving with intelligent vehicles: Driving behavior with ACC and the acceptance by individual drivers. 2000 IEEE Intelligent Transportation Systems, Oct 1-2, 506-9.

Lee, J.D., McGehee, D.V., Brown, T.L., \& Reyes, M.L. (2002). Collision warning timing, driver distraction, and driver response to imminent rear-end collisions in a high-fidelity driving simulator. Human Factors, 44(2): 303-314.

Lee, J. D., \& See, K. A. (2004). Trust in technology: Designing for appropriate reliance. Human Factors, 46(1): 50-80.

National Center for Statistics and Analysis. Traffic Safety Facts 2003. Retrieved December 12, 2004 from http://www-nrd.nhtsa.dot.gov/pdf/nrd-30/NCSA/TSF2003/809767.pdf

Nilsson, L. (1995). Safety effects of adaptive cruise control in critical traffic situations, Proceedings of the Second World Congress on Intelligent Transport Systems: 'Steps Forward', Vol. III, (Yokohama: VERTIS), 1254-1259.

Stanton, N.A. \& Marsden, P. (1996). From fly-by-wire to drive-by-wire: Safety implications of automation in vehicles. Safety Science, 24(1): 35-49.

Stanton, N.A. \& Young, M.S. (1998). Vehicle automation and driving performance. Ergonomics, 41(7): 1014-1028.

Young, M.S. \& Stanton, N.A. (2002). Malleable attentional resources theory: A new explanation for the effects of mental underload on performance. Human Factors, 44(3): 365-375. 